\title{
Expression of Concern: shRNA-mediated silencing of ZFX attenuated the proliferation of breast cancer cells
}

\author{
Hongjian Yang ${ }^{1} \cdot$ Yue $\mathrm{Lu}^{2}$. Yabing Zheng ${ }^{3} \cdot$ Xingfei Yu $^{1} \cdot$ Xianghou Xia $^{1} \cdot$ Xiangming He$^{1} \cdot$ Weiliang Feng $^{1} \cdot$ Lei Xing $^{1}$. \\ Zhiqiang Ling ${ }^{4}$
}

Published online: 10 April 2020

(c) Springer-Verlag GmbH Germany, part of Springer Nature 2020

\section{Expression of Concern: \\ Cancer Chemother Pharmacol (2014) 73:569-576 \\ https://doi.org/10.1007/s00280-014-2379-y}

The Editors-in-Chief would like to alert readers that there are some concerns related to this article [1]. Concern has been raised that there are textual and formatting similarities to this retracted article [2]. There are also concerns about the construction of experimental reagents in the methods, as well as in the phrases selected for reporting cell cycle influences. While we recognize that the overlap could result from recycling of language with similar experimental methodologies, particularly by authors whose first language is not English, we have not received any response from the authors regarding the above concerns. None of the authors have responded to correspondence about this Expression of Concern. Readers are advised to interpret the details of this article with caution.

\section{References}

1. Yang H, Lu Y, Zheng Y et al (2014) Cancer Chemother Pharmacol 73:569. https://doi.org/10.1007/s00280-014-2379-y

2. Huang WY, Chen DH, Ning L, Wang LW (2012) Asian Pac J Cancer Prev 13(5):1823-1827. https://doi.org/10.22034/APJCP .2017.18.10.2891

Publisher's Note Springer Nature remains neutral with regard to jurisdictional claims in published maps and institutional affiliations.
The original article can be found online at https://doi.org/10.1007/ s00280-014-2379-y.

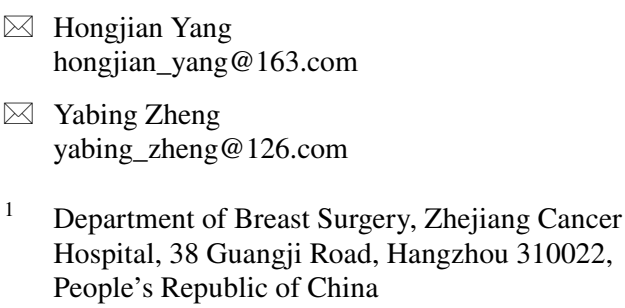

2 Cancer Center, The Medical School, Hospital of Qingdao University, No. 16, Jiangsu Road, Qingdao 266003, People's Republic of China

3 Department of Internal Oncology, Zhejiang Cancer Hospital, 38 Guangji Road, Hangzhou 310022, People's Republic of China

4 Zhejiang Cancer Research Institute, Zhejiang Cancer Hospital, 38 Guangji Road, Hangzhou 310022, People's Republic of China 